\title{
M-Budget oder dass die Medizin nicht zum Konsumgut wird
}

\section{Remo Osterwalder}

Dr. med., Vizepräsident der FMH, Departementsverantwortlicher Dienstleistungen und Berufsentwicklung

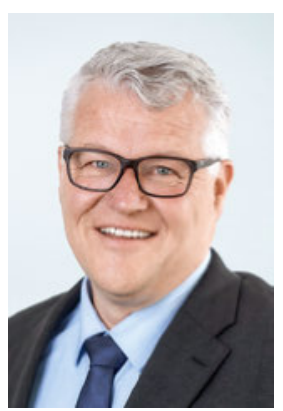

So wie die Medizin in ständigem Wandel steht, verändert sich auch die Versorgungslandschaft der Anbieter medizinischer Leistungen. War früher noch das altbekannte Hausarztmodell mit der Einzelpraxis, mussten wir inzwischen feststellen, dass dieses Modell für die Ausübung des Arztberufs nicht mehr attraktiv genug war. Stattdessen sind die Gruppenpraxen als Alternative aufgekommen. Aber auch sie konnten den Bedarf bis heute nicht ausreichend decken, wenn es um die Grundversorgung geht. Als weitere Praxisform gibt es die Gesundheitszentren mit interdisziplinären Leistungen. Die Gefahr besteht dort, dass aus verschiedenen Gründen die Kosten pro Patient im Schnitt höher sein können als bei reinen Grundversorger-Gruppen-

\section{Erfolgsmodelle hängen vom Goodwill der Patienten ab.}

praxen. Bislang fehlen aber Qualitätsindikatoren, um zu sehen, ob auch die Hospitalisationrate zum Beispiel in diesen Vergleichskollektiven gleich ist oder ob nur die reinen Grundleistungen, also die direkten Kosten, verglichen werden.

Nun gibt es eine richtige Welle von Praxiseröffnungen durch Interessenten mit unterschiedlicher Motivation. Die Spitäler wollen ihre Zuweisungen im Einzugsgebiet sichern durch den Aufkauf von Praxen und die Besetzung mit ihrem Personal. Aufgrund der Effizienzrate ist dies auf die Einzelpraxis bezogen nicht immer kostendeckend, wenn die indirekte Wirkung durch die stationären Fälle oder die Abklärungen durch die spitalinternen Spezialkliniken nicht berücksichtigt werden. Aber auch andere Interessenten wie die Versicherer drangen in den Gesundheitsmarkt ein. Der Wille, die Kosten in den Griff zu bekommen, ist nicht nur auf die Ärztinnen und Ärzte beschränkt. Es scheint, dass auch die Versicherer sich das Abenteuer Arztpraxis anders vorgestellt haben, sonst lässt sich ihre Strategieänderung mit dem Verkauf der Praxen nicht erklären.
Jetzt kommt eine ganz andere Art von Teilnehmer auf den Markt: die Migros. Der orange Riese ist ja schon mit seinen Fitnesszentren auf dem Gesundheitsmarkt präsent. So wie Gottlieb Duttweiler nach dem Zusammenbruch seiner ursprünglichen Firma 1920 (Pfister \& Duttweiler) mit den Verkaufswagen der neuen Firma Migros den Detailhandel von Grund auf veränderte, könnte auch im Gesundheitsmarkt eine neue Epoche beginnen. Eine wichtige Grundvoraussetzung für jede medizinische Behandlung ist die Vertrauensbasis zwischen $\mathrm{Pa}$ tient und Ärztin respektive Arzt. Hier hat zum Beispiel das System Cumulus wenig Platz, wenn es darum geht, die Marketingausrichtung unter dem Deckmantel der personalisierten Medizin voranzutreiben. Anders gesagt: Das Sammeln von persönlichen Gesundheitsdaten darf nur dann angewandt werden, wenn es der direkten Förderung der Gesundheit des Patienten dient und keinen kommerziellen Hintergrund hat.

Betrachten wir doch kurz die Realität im täglichen Gesundheitsgeschäft: Der Löwenanteil der Gesundheitskosten wird durch eine Minderheit der Patienten während eines relativ kurzen Lebensabschnitts verursacht. Gerade diese Klientel stellt eine enorme Herausforde-

\section{In der Arzt-Patient-Beziehung hat das Cumulus-} System wenig Platz.

rung an die zukünftige Versorgungslandschaft. Chronisch Kranke wünschen mehrheitlich eine Bezugsperson als Referenz, wobei es sich nicht immer um eine Ärztin oder einen Arzt handeln muss. Gerade hier tun sich enorme Perspektiven für die Interprofessionalität auf. Wichtig ist für die Patienten, dass die Bezugsperson nicht ständig wechselt und eine kohärente Behandlungsstrategie verfolgt wird. Ein Erfolgsmodell hängt stark ab vom Vertrauen der Patienten und der Motivation aller, die an der Behandlung beteiligt sind. Lassen wir uns überraschen, ob sich das Erfolgsmodell von Gottlieb Duttweiler auch auf den schweizerischen Gesundheitsmarkt übertragen lässt. 